\title{
Nonlinear Implementable Control of a Dual Active Bridge Series Resonant Converter
}

\author{
Eduardo Sebastián, Eduardo Montijano, Estanis Oyarbide, Carlos Bernal, Rubén Gálvez
}

\begin{abstract}
This paper presents a novel control strategy for a Dual Active Bridge Series Resonant Converter (DABSRC). The strategy seeks to ensure the stability of the converter over its entire dynamic range while enhances the transient response. Both properties allow the use of the converter in new applications where fast dynamics are required, surpassing the performance of existing feedback loops. Starting from the generalized averaged model of the converter, we propose a nonlinear control strategy by means of Lyapunov's stability theory. After that, we derive a series of modifications in order to implement the strategy in a microcontroller or a DSP, including a sensorless method to tackle the lack of measurements of certain variables and an adaptive control law to deal with uncertain parameters in the model. The strategy is evaluated in simulations and experiments, employing a commercial converter and comparing the results with other control policies.
\end{abstract}

Index Terms-DC-DC power converters, nonlinear control systems, resonant converters, switching converters, sensorless control

\section{INTRODUCTION}

$\mathbf{P}$ OWER electronics is present in many of today's technological advances. In particular, bi-directional DC/DC resonant converters are an efficient option when high voltage and power conversion ratios are required [1], enabling applications with fast and changing dynamics [2]. For example, in an energy-recovery system for elevator retrofit [3], when the energy recovered by the motor-inverter system makes the voltage of the DC bus to increase, a DC/DC converter evacuates this extra energy to a supercapacitor bank. On the other hand, when the voltage of the DC bus capacitor decreases, the DC/DC must inject the required energy. Moreover, this must be done minimizing the number of sensors and the computational capacity of the digital devices running the control [4]. Another application example can be found on automated guided vehicles (AGV), where a DC/DC converter links the low voltage $(48 \mathrm{~V})$ battery with the industrial level voltage $(600 \mathrm{~V}) \mathrm{DC}$ bus of the inverter. Due to the small value of the DC bus capacitor, fast load steps lead to deep

This work was supported by project DGA T04-FSE and Spanish grant FPU19-05700. We are grateful for this support.

E. Sebastián and E. Montijano are with the Dept. of Computer Science and Systems Engineering at University of Zaragoza, Spain (e-mail: esebastian@unizar.es, emonti@unizar.es).

E. Oyarbide and C. Bernal are with the Dept. of Electronics Engineering and Communication at University of Zaragoza, Spain (e-mail: eoyarbid@unizar.es, cbernal@unizar.es).

R. Gálvez is with Epic Power Converters, Spain (e-mail: rgalvez@epicpower.es). voltage variations at the bus capacitor. The DC/DC converter must exhibit a fast control response. Similar conclusions can be derived from other applications such as renewable energy stations [5] or the electric car [6]. A popular topology in this kind of applications is the Dual Active Bridge (DAB) [7], [8] due to its low stress and small filter components. When low switching losses are required, the best option is to include a Series Resonant Converter (SRC) [9]-[11]. All the aforementioned features make the control of the DAB-SRC [12], [13] specially challenging, evidenced in the absence of advanced controllers on the DAB-SRC in the literature. In this context, this paper copes with the control of a DAB-SRC to enhance its performance, proposing a robust nonlinear control law implementable in a commercial converter.

A common practice in the control of complex converters is the use of nested feedback loops [14] based on linear techniques. This is the case of [15] and [16] for the DAB. Indeed, the use of linear regulators is the standard in several industrial applications [17] because they are simple to tune and enough for a stable and robust performance, at the expense of slowing down the system to reject perturbations and nonlinear effects. The resulting poor transient dynamic is not a problem in many applications, e.g., when charging batteries. However, there are other applications where the transient response of the $\mathrm{DC} / \mathrm{DC}$ converter is critical, as it is the case of regulating the voltage of the DC bus capacitor of an inverter. In this case, the DC/DC converter must react rapidly in order to avoid strong voltage variations at the $\mathrm{DC}$ bus capacitor. A DAB converter with a PI controller exhibits a fast response but in the case of a DAB-SRC converter, its strong nonlinearities and resonance behavior makes almost impossible to get a fast response based on linear converters. Thus, we aim at considering all nonlinear effects to enhance both speed and robustness.

In the search of faster and more robust control solutions, several works employ nonlinear control techniques. An instance is [18], where robust control is used to ensure the stability of a Buck against uncertain parameters. Lyapunov's stability theory is the base of [19] to develop a passive, adaptive control in a single-phase inverter. Compared to them, we deal with a more complex topology due to the DAB topology and the SRC circuit. A well-known technique to regulate power converters is the Sliding Mode Control [20], approach taken by [21] in a DAB converter. Nevertheless, this approach requires high control frequency and precise knowledge of the parameters to perfectly compensate the nonlinear dynamics.

There are two other control alternatives with interesting 
properties. The first one is the deadbeat control [22]-[24], which obtains the fastest response in simple topologies [22] and can be integrated in optimal controllers [23]. The second alternative is State Trajectory Control (STC) [25], [26]. The seminal work in [27] proposes a simplification which enables its implementation in an $L L C$ converter. However, both methodologies, deadbeat and STC, require the control frequency to be similar to the switching frequency, which in the converter used in our experiments is not possible because the control frequency is 15 to 40 times slower than the switching frequency.

Therefore, the main contribution of this paper is a novel control strategy for a DAB-SRC that guarantees global stability and a fast transient response in any topologically reachable configuration. These properties have a favorable impact on power electronics applications, as they ensure the correct operation of the converter in flexible and fast scenarios. The other contribution of this work is a series of adaptations to enhance the robustness of the control and enable its implementation in a commercial device.

The rest of the article is as follows. Section II presents the topology of the converter. Then, Section III describes the control strategy. The implementation of the proposal requires some modifications, derived in Section IV. The control proposal is simulated in Section V whereas Section VI includes the experimental results in a commercial converter. Finally, Section VII is the conclusion.

\section{MODEL DESCRIPTION}

The converter under study is shown in Fig. 1. There are two full bridges interconnected by an $L C$ series resonant tank and a transformer. In this case $V_{a}$ is the high voltage side and $V_{o}$ corresponds with the low voltage side. Parameter $L$ models both the resonant inductance and the leakage inductances of the high frequency transformer. A resistor $R$ includes power losses at the resonant tank, transformer and semiconductors.

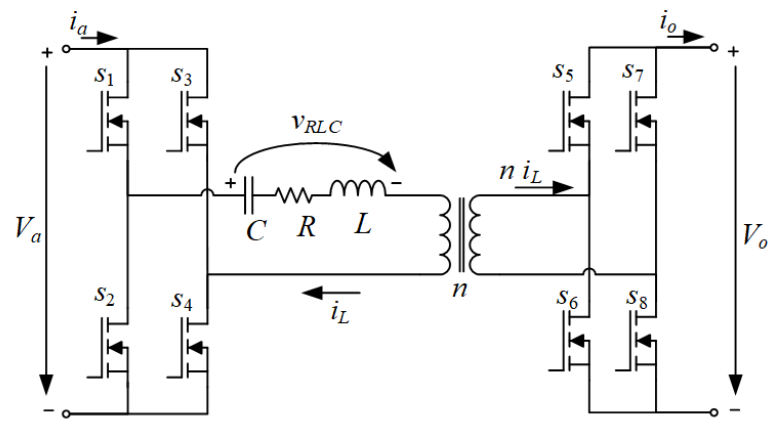

Fig. 1. DAB-SRC under study.

One of the bridges works as an inverter and the other as a rectifier. In the example shown in this work the power flows from $V_{a}$ to $V_{o}$. Fig. 2 is the transformer-left-side equivalent circuit of the converter, where $V_{b}=n V_{o}$, with $n$ the transformer turns ratio.

Signals $u_{1}, u_{2} \in\{-1,1\}$ are switching variables in such a way that S1 and S4 (S5 and S8) are on if $u_{1}=1\left(u_{2}=1\right)$

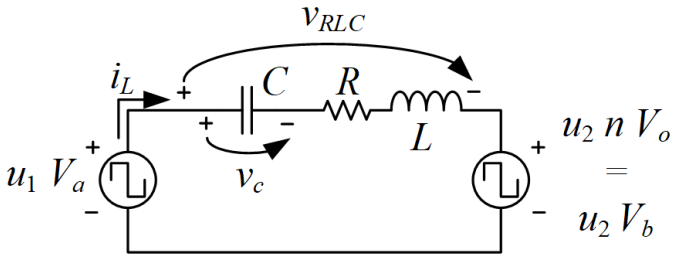

Fig. 2. Equivalent circuit of the DAB-SRC under study.

whereas S2 and S3 (S6 and S7) are on if $u_{1}=-1\left(u_{2}=\right.$ $-1)$. Fig. 3 shows the most relevant waveforms on steadystate operation. A full wave modulation is applied at each bridge $\left(u_{1} V_{a}\right.$ and $\left.u_{2} V_{b}\right)$, being $\delta$ the phase shift between the output waves of both bridges. As the switching frequency is above the resonant frequency ZVS operation is assured and therefore only turn-off losses must be considered, i.e., losses due to $i_{\text {toffa }}$ at the high-side bridge and caused by $i_{\text {toffb }}$ at the low-voltage side.

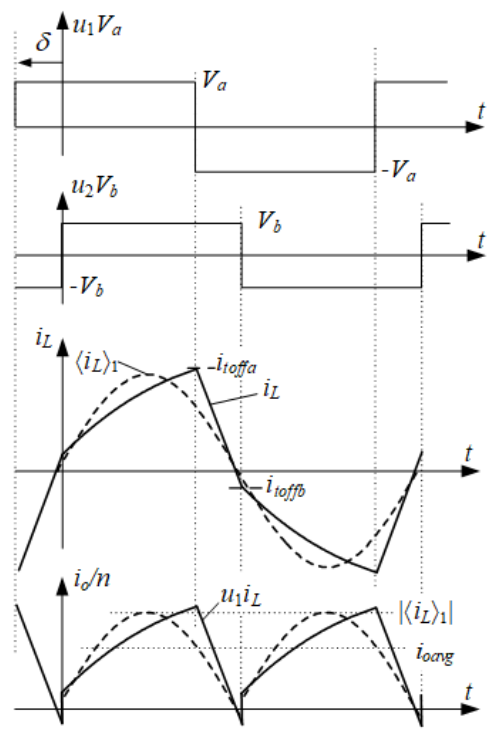

Fig. 3. Significant waveforms.

The switching frequency $w$ (shared by the two bridges) and the phase shift $\delta$ are the control variables. The topological model of the converter is represented by Eqs. (1)-(2)

$$
\begin{aligned}
\frac{d i_{L}}{d t} & =\frac{1}{L}\left(u_{1} V_{a}-R i_{L}-v_{C}-u_{2} V_{b}\right) \\
\frac{d v_{C}}{d t} & =\frac{i_{L}}{C}
\end{aligned}
$$

Applying the Generalized Averaging Method [28] [29] up to the first harmonic, it yields to (3)-(4)

$$
\frac{d\left\langle i_{L}\right\rangle_{1}}{d t}=\frac{\left\langle u_{1}\right\rangle_{1} V_{a}}{L}-\frac{R\left\langle i_{L}\right\rangle_{1}}{L}-\frac{\left\langle v_{C}\right\rangle_{1}}{L}-\frac{\left\langle u_{2}\right\rangle_{1} V_{b}}{L}-j w\left\langle i_{L}\right\rangle_{1}
$$

$\frac{d\left\langle v_{C}\right\rangle_{1}}{d t}=\frac{\left\langle i_{L}\right\rangle_{1}}{C}-j w\left\langle v_{C}\right\rangle_{1}$ 
Considering the phase of the output modulation wave $u_{2}$ as the phase reference, the next variable changes are possible

$$
\begin{aligned}
\left\langle i_{L}\right\rangle_{1} & =i_{L R}+j i_{L I} & \left\langle v_{C}\right\rangle_{1} & =v_{C R} \\
\left\langle u_{1}\right\rangle_{1} & =\frac{2}{\pi} \sin (\delta)-j \frac{2}{\pi} \cos (\delta) & \left\langle u_{2}\right\rangle_{1} & =-j \frac{2}{\pi}
\end{aligned}
$$

where $i_{L R}$ and $i_{L I}$ (resp. $v_{C R}$ and $v_{C I}$ ) are the real and imaginary part of $i_{L}$ (resp. $\left.v_{C}\right)$.

Finally, the model of the converter is described by

$$
\begin{aligned}
& \frac{d i_{L R}}{d t}=-\frac{R i_{L R}}{L}+w i_{L I}-\frac{v_{C R}}{L}+\frac{2 V_{a}}{L \pi} \sin (\delta), \\
& \frac{d i_{L I}}{d t}=-w i_{L R}-\frac{R i_{L I}}{L}-\frac{v_{C I}}{L}+\frac{2 V_{b}}{L \pi}-\frac{2 V_{a}}{L \pi} \cos (\delta), \\
& \frac{d v_{C R}}{d t}=\frac{i_{L R}}{C}+w v_{C I}, \\
& \frac{d v_{C I}}{d t}=\frac{i_{L I}}{C}-w v_{C R},
\end{aligned}
$$

where $i_{L R}, i_{L I}$ are the controlled variables, and $v_{C R}, v_{C I}$ are the so-called free variables. The selection of this controlled variables provides both flexibility and generality in the proposal, allowing the practitioner to map in them any desired application requirement regarding the power conversion, efficiency and technological requests. The control variables are the switching frequency, $w$, which is normally related to the converted power, and the phase shift $\delta$, which has a great influence in the turn-off current. As it can be observed in Eqs. (5)-(8), the control variables drive the dynamics of the controlled variables through strongly nonlinear equations. Therefore, conventional control strategies as PI controllers are not able to provide fast and stable transients.

\section{CONTROL LAW}

This Section describes the control proposal, main contribution of this paper. In order to adequate to the control notation, let $\mathbf{x}$ denote the state vector and $\mathbf{u}$ the action vector,

$$
\begin{aligned}
& \mathbf{x}=\left[\begin{array}{llll}
x_{1} & x_{2} & x_{3} & x_{4}
\end{array}\right]^{T}=\left[\begin{array}{llll}
i_{L R} & i_{L I} & v_{C R} & v_{C I}
\end{array}\right]^{T} \\
& \mathbf{u}=\left[\begin{array}{ll}
\delta & w
\end{array}\right]^{T} .
\end{aligned}
$$

The goal is to drive the converter to a desired configuration, denoted by $\mathbf{x}^{*}$, so that the control error is $\widetilde{\mathbf{x}}=\mathbf{x}-\mathbf{x}^{*}$. According to the description of the model in Section II, $x_{1}$ and $x_{2}$ are the controlled states, so $x_{3}^{*}=x_{3}(t)$ and $x_{4}^{*}=x_{4}(t)$ for all $t$. Recalling our objectives, we seek an expression for $\mathbf{u}$ to achieve stability of the converter $\forall \mathbf{x}$.

To this end, in this paper we propose a nonlinear control strategy based on two types of controllers. The intuition is the following. When the converter is far from the desired state, we apply nonlinear control expressions in $\delta$ and $w$ which achieve a fast initial transient response and small error. Near the desired configuration we also include in the control law two PIs, one for $\delta$ and the other for $w$, to ensure a smooth convergence of the converter to the desired configuration. The control expressions are described by

$$
\begin{aligned}
& \delta= \begin{cases}\arctan 2\left(K_{2} \tilde{x}_{2}, K_{1} \tilde{x}_{1}\right), \quad \text { if } \tau>\varepsilon \\
K_{P}^{\delta} \tilde{x}_{1}+K_{I}^{\delta} \int_{0}^{t} \tilde{x}_{1} d t, \quad \text { otherwise }\end{cases} \\
& w=\left\{\begin{array}{cc}
\frac{\frac{1}{L} K_{1} x_{3} \tilde{x}_{1}-\frac{R}{L} K_{1} x_{1} x_{1}^{*}+\frac{1}{L} K_{2} x_{4} \widetilde{x}_{2}}{K_{1} x_{2} \tilde{x}_{1}-K_{2} x_{1} \tilde{x}_{2}} \\
+\frac{-\frac{2 V_{b}}{L \pi} K_{2} \widetilde{x}_{2}-\frac{R}{L} K_{2} x_{2} x_{2}^{*}}{K_{1} x_{2} \tilde{x}_{1}-K_{2} x_{1} \tilde{x}_{2}}, & \text { if } \tau>\varepsilon \\
K_{P}^{w} \tilde{x}_{2}+K_{I}^{w} \int_{0}^{t} \tilde{x}_{2} d t, & \text { otherwise }
\end{array}\right.
\end{aligned}
$$

with $K_{1}, K_{2}, K_{P}^{\delta}, K_{I}^{\delta}, K_{P}^{w}, K_{I}^{w}$ and $\varepsilon$ positive constants and

$$
\tau=\left|\frac{\tilde{x}_{2}}{x_{2}}\right| .
$$

The inclusion of the PIs is required because the nonlinear control law for $w$ has a division by zero in the desired configuration, i.e., when $\tilde{x}_{1}=\tilde{x}_{2}=0$ the denominator in (10), $K_{1} x_{2} \tilde{x}_{1}-K_{2} x_{1} \tilde{x}_{2}$, is zero. From an application point of view, this also becomes a problem near $\mathrm{x}^{*}$ because small changes in the error result in big changes in the action $w$. The transition between the nonlinear expression and the PIs is done in such a way that the action preserves continuity, initializing the integral terms by

$$
\begin{aligned}
& \int_{0}^{t} \tilde{x}_{1} d t=\left(\delta_{L y}-K_{P}^{\delta} \tilde{x}_{1}\right) / K_{I}^{\delta} \\
& \int_{0}^{t} \tilde{x}_{2} d t=\left(w_{L y}-K_{P}^{w} \tilde{x}_{2}\right) / K_{I}^{w}
\end{aligned}
$$

with $\delta_{L y}$ and $w_{L y}$ the actions from the nonlinear terms when the transition occurs. The parameter $\varepsilon$ defines the transition moment. On one hand it needs to be big to prevent undesired behaviors of the nonlinear term in (10) near the singularity. On the other hand, since the model is nonlinear, the PIs will only stabilize the system if the state is sufficiently close to the desired configuration, which requires a small value of $\varepsilon$. The following proposition demonstrates that there is always a sufficiently small value of $\varepsilon$ that formally guarantees the global and asymptotic stability (GAS) of the converter.

Proposition 1. Let $K_{P}^{\delta}, K_{I}^{\delta}, K_{P}^{w}, K_{I}^{w}$ be some constants such that $\widetilde{\mathbf{x}}=\mathbf{0}$ is a stable point of the linearized system in (5)-(8) under the PI control. Then, there exists a sufficiently small $\epsilon$ such that the control strategy of Eqs. (9)-(10) is GAS.

Proof. Let $V(\tilde{\mathbf{x}})=\frac{1}{2} K_{1} \tilde{x}_{1}^{2}+\frac{1}{2} K_{2} \tilde{x}_{2}^{2} \in \mathbb{R}$ be a Lyapunov function candidate. By developing $\dot{V}$ it follows that

$$
\begin{aligned}
\dot{V}(\tilde{\mathbf{x}})= & K_{1} \tilde{x}_{1} \dot{\tilde{x}}_{1}+K_{2} \tilde{x}_{2} \dot{\tilde{x}}_{2}= \\
& K_{1} \tilde{x}_{1} \dot{x}_{1}+K_{2} \tilde{x}_{2} \dot{x}_{2}= \\
& w K_{1} x_{2} \widetilde{x}_{1}-\frac{R}{L} K_{1} x_{1}^{2}-\frac{1}{L} K_{1} x_{3} \widetilde{x}_{1}+\frac{R}{L} K_{1} x_{1} x_{1}^{*}- \\
& -w K_{2} x_{1} \widetilde{x}_{2}-\frac{R}{L} K_{2} x_{2}^{2}-\frac{1}{L} K_{2} x_{4} \widetilde{x}_{2}+\frac{2 V_{b}}{L \pi} K_{2} \widetilde{x}_{2}+ \\
& \frac{R}{L} K_{2} x_{2} x_{2}^{*}+K_{1} \tilde{x}_{1} \frac{2 V_{a}}{L \pi} \sin \delta-K_{2} \tilde{x}_{2} \frac{2 V_{a}}{L \pi} \cos \delta .
\end{aligned}
$$


If $\tau>\varepsilon$, substitution of Eqs. (9) and (10) in Eq. (14) gives

$$
\dot{V}(\widetilde{\mathbf{x}})=-\frac{R}{L} K_{1} x_{1}^{2}-\frac{R}{L} K_{2} x_{2}^{2},
$$

which is negative $\forall \widetilde{\mathbf{x}}$. This means that from any initial conditions, there will be a time such that $\tau \leq \varepsilon$, forcing the switch to the PI controllers. The initialization in (12)-(13) ensures continuity of the action. By assumption in the claim, the PIs are designed such that the linearized system is stable in $\widetilde{\mathbf{x}}=\mathbf{0}$. This implies [30] that there exists a neighborhood $D$ of $\widetilde{\mathbf{x}}=\mathbf{0}$ where the PIs achieve local asymptotic stability of the nonlinear system. Thus, there exists a sufficiently small $\varepsilon$ such that the control achieves GAS, concluding the proof.

The only missing part is the choice of an appropriate value for $\varepsilon$. This could be tackled by means of simulations and optimization search over the state space of the converter. Nonetheless, in practice it is just enough to set $\varepsilon$ to a sufficiently small value, e.g., 0.05 in our experiments, to obtain a stable and fast response even with an aggressive PI design.

\section{AdAPTATIONS}

In an ideal scenario, this proposal is ready to control the converter. However, some issues arise from the implementation requirements.

Firstly, the control strategy depends on $x_{3}$ and $x_{4}$. In particular, this dependence is represented in Eq. (14) by

$$
-\frac{1}{L} K_{1} x_{3} \tilde{x}_{1}-\frac{1}{L} K_{2} x_{4} \tilde{x}_{2} .
$$

However, this information is not accessible in the real system since $x_{3}$ and $x_{4}$ correspond to the real and imaginary part of $v_{C}$. As we can not measure them, a sensorless method is proposed which eliminates this dependence.

Considering the limitations in the design of power converters, the voltage at the resonance capacitor $v_{C}$ is always below a certain value $V_{l i m}$. Recalling the harmonic approximation in Section II,

$$
v_{C, p p}=\sqrt{2} v_{C, r m s}=\sqrt{x_{3}^{2}+x_{4}^{2}},
$$

and so both $x_{3}$ and $x_{4}$ are limited by the maximum admissible voltage in the capacitor

$v_{C, p p}=\sqrt{2} v_{C, r m s}=\sqrt{x_{3}^{2}+x_{4}^{2}} \leq V_{\text {lim }} \rightarrow\left|x_{3}\right|,\left|x_{4}\right| \leq V_{\text {lim }}$.

In the worst case, that is, Eq. (16) is at its maximum value, the term

$$
\frac{1}{L} V_{\text {lim }}\left(K_{1}\left|\tilde{x}_{1}\right|+K_{2}\left|\tilde{x}_{2}\right|\right)
$$

must be compensated. The modification consists in replacing (16) by (19) in the nonlinear term of (10). Additionally to the sensorless feature, this adaptation has the advantage that it only adds speed to the transient response since the numerator in Eq. (10) when $\tau>\varepsilon$ increases. Finally, the value of $C$ is no longer required, so the control is robust to any uncertainty or tolerance with respect to the resonance capacitor.

The second modification is related to the difference between the characterized parameters and the real ones. This could lead to an incorrect compensation of the terms in (9) and (10) and destabilize the system. To overcome this circumstance, we propose an adaptive law which works in real time. Let us define $a_{1}=\frac{R}{L}$ and $a_{2}=\frac{1}{L}$ to ease the design. These parameters correspond to the coefficients multiplying the terms in the model (5)-(8). In case $R$ and $L$ are not known with prevision, $a_{1}$ and $a_{2}$ can be expressed as the sum of two terms $a_{1}=\hat{a}_{1}+\tilde{a}_{1}$ and $a_{2}=\hat{a}_{2}+\tilde{a}_{2}$. Here, $\hat{a}_{1}$ and $\hat{a}_{2}$ are the estimated parameters whilst $\tilde{a}_{1}$ and $\tilde{a}_{2}$ are the error between the real and estimated parameters.

The adaptation law seeks to ensure the stability of the converter by evolving the values $\tilde{a}_{1}$ and $\tilde{a}_{2}$, in such a way that the control defined in Eqs. (9) and (10) still works. So the condition of negative derivative over $\dot{V}$ must be preserved.

Proposition 2. Given the control law defined by Eqs. (9)(10) and the design conditions described in Proposition 1, the adaptation law

$$
\begin{aligned}
& \dot{\hat{a}}_{1}=-\frac{1}{K_{a_{1}}}\left(K_{1} x_{1} \tilde{x}_{1}+K_{2} x_{2} \tilde{x}_{2}\right) \\
& \dot{\hat{a}}_{2}=-\frac{1}{K_{a_{2}}}\left(K_{1} V_{\text {lim }} \tilde{x}_{1}+K_{2} V_{\text {lim }} \tilde{x}_{2}-\frac{2 V_{b}}{\pi} K_{2} \tilde{x}_{2}\right)
\end{aligned}
$$

preserves the GAS, with $K_{a_{1}}$ and $K_{a_{2}}$ two positive gains.

Proof. Redefine the Lyapunov function in Proposition 1 to consider the error in the parameters

$$
V\left(\tilde{\mathbf{x}}, \tilde{a}_{1}, \tilde{a}_{2}\right)=\frac{1}{2} K_{1} \tilde{x}_{1}^{2}+\frac{1}{2} K_{2} \tilde{x}_{2}^{2}+\frac{1}{2} K_{a_{1}} \tilde{a}_{1}^{2}+\frac{1}{2} K_{a_{2}} \tilde{a}_{2}^{2} .
$$

Then, $\dot{V}$ changes to

$$
\begin{aligned}
\dot{V}\left(\tilde{\mathbf{x}}, \tilde{a}_{1}, \tilde{a}_{2}\right)= & K_{1} \tilde{x}_{1} \dot{x}_{1}+K_{2} \tilde{x}_{2} \dot{x}_{2}+K_{a_{1}} \tilde{a}_{1} \dot{\tilde{a}}_{1}+K_{a_{2}} \tilde{a}_{2} \dot{\tilde{a}}_{2}= \\
& K_{a_{1}} \tilde{a}_{1} \dot{\tilde{a}}_{1}+K_{a_{2}} \tilde{a}_{2} \dot{\tilde{a}}_{2}-\tilde{a}_{1} K_{1} x_{1} \tilde{x}_{1}-\tilde{a}_{2} K_{1} x_{3} \tilde{x}_{1} \\
& +\tilde{a}_{2} K_{1} \tilde{x}_{1} \frac{2 V_{a}}{\pi} \sin \delta-\tilde{a}_{1} K_{2} x_{2} \tilde{x}_{2}-\tilde{a}_{2} K_{2} x_{4} \tilde{x}_{2} \\
& +\tilde{a}_{2} \frac{2 V_{b}}{\pi} K_{2} \tilde{x}_{2}-\tilde{a}_{2} K_{2} \tilde{x}_{2} \frac{2 V_{a}}{\pi} \cos \delta+W
\end{aligned}
$$

where $W$ includes all the terms which depend on $\hat{a}_{1}$ and $\hat{a}_{2}$ and it is strictly negative, since is the result of applying the control law in Eqs. (9) and (10). To preserve the GAS properties over $\dot{V}$, the other terms are distributed

$$
\begin{aligned}
& K_{a_{1}} \tilde{a}_{1} \dot{\tilde{a}}_{1}=\tilde{a}_{1} K_{1} x_{1} \tilde{x}_{1}+\tilde{a}_{1} K_{2} x_{2} \tilde{x}_{2}, \\
& K_{a_{2}} \tilde{a}_{2} \dot{\tilde{a}}_{2}=\tilde{a}_{2} K_{1} x_{3} \tilde{x}_{1}+\tilde{a}_{2} K_{2} x_{4} \tilde{x}_{2}-\tilde{a}_{2} \frac{2 V_{b}}{\pi} K_{2} \tilde{x}_{2},
\end{aligned}
$$

considering that $\tilde{a}_{2} K_{1} \tilde{x}_{1} \frac{2 V_{a}}{\pi} \sin \delta=\tilde{a}_{2} K_{2} \tilde{x}_{2} \frac{2 V_{a}}{\pi} \cos \delta$ by the definition of $\delta$. Then,

$$
\begin{aligned}
& \dot{\tilde{a}}_{1}=\frac{1}{K_{a_{1}}}\left(K_{1} x_{1} \tilde{x}_{1}+K_{2} x_{2} \tilde{x}_{2}\right), \\
& \dot{\tilde{a}}_{2}=\frac{1}{K_{a_{2}}}\left(K_{1} x_{3} \tilde{x}_{1}+K_{2} x_{4} \tilde{x}_{2}-\frac{2 V_{b}}{\pi} K_{2} \tilde{x}_{2}\right) .
\end{aligned}
$$

Assuming that the real parameters are quasi-static

$$
\dot{\hat{a}}_{1}=-\dot{\tilde{a}}_{1}, \quad \quad \dot{\hat{a}}_{2}=-\dot{\tilde{a}}_{2} .
$$


The sensorless term in (19) is included in Eq. (21) to consider the absence of measurements in $x_{3}$ and $x_{4}$. Additionally, Eq. (10) changes to

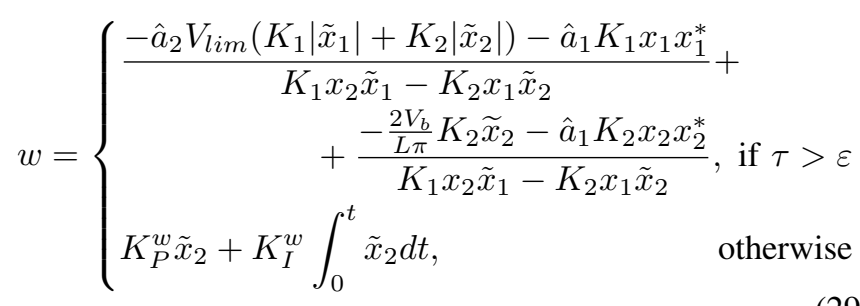

At this point, following a similar procedure to the one described in the proof of Proposition 1, GAS is proved.

It is underlined that the adaptation law does not seek to obtain the precise real value of the parameters. It simply evolves the estimates to ensure the stability of the converter.

The last aspect to address is the discretization of the control strategy. Its implementation is done by a digital device, which immediately implies that the control strategy must work in discrete time with a sampling time $T_{s}$. This contrast with the continuous time control proposal. Aiming to preserve the properties and structure of the nonlinear controller, a limitation in the velocity of the actions is considered,

$$
|\dot{\delta}|<\dot{\delta}_{\max } \quad|\dot{w}|<\dot{w}_{\max }
$$

with $\dot{\delta}_{\max }$ and $\dot{w}_{\max }$ part of the design. Thanks to this, the control strategy can be implemented in discrete time by a Zero Order Hold $(\mathrm{ZOH})$. By limiting the change in $\mathbf{u}$, the behavior of the converter is smoothed out because the overdamped dynamic dominates the underdamped dynamic characteristic of the resonant circuit. The speed of the transient response is not deteriorated because the control strategy pursues to control the overdamped dynamic. Furthermore, by limiting $|\dot{\delta}|$ and $|\dot{w}|$ the devices are being protected from premature degradation.

Algorithm 1 summarizes the sequence of control steps of the complete strategy, and Fig. 4 details the design process for the control parameters. Constants $K_{1}$ and $K_{2}$ are tuned according to the desired settling time of $x_{1}$ and $x_{2}$ respectively, since they are multiplied by the corresponding errors in Eq. (29). Then, we obtain the linearized model in Eqs. (5)-(8) to design $K_{P}^{\delta}, K_{I}^{\delta}, K_{P}^{w}, K_{I}^{w}$ using pole placement, to ensure stability in the small gain. Nevertheless, any other classic technique can be applied for this step and, if needed, it can be further refined in experiments. The transient response is finally tuned by $K_{a_{1}}$ and $K_{a_{2}}$, which permit us to speed up $x_{1}$ and $x_{2}$ since they drive the evolution of $\hat{a}_{1}$ and $\hat{a}_{2}$. The selection of $\varepsilon$ is empirical and depends on the model of the DABSRC: it must be sufficiently small to leverage the speed of the nonlinear controller and big enough to satisfy stability and obtain zero steady-state error. Finally, $\dot{\delta}_{\max }, \dot{w}_{\max }$ are selected to verify security, sampling time and other constraints on the real device.

Note that all expressions can be implemented in a digital device. The only operation that might not be available is the arc tangent, but in that case it could be implemented through a LUT. The design formally guarantees the stability of the converter and, therefore, zero error in the steady state. On the

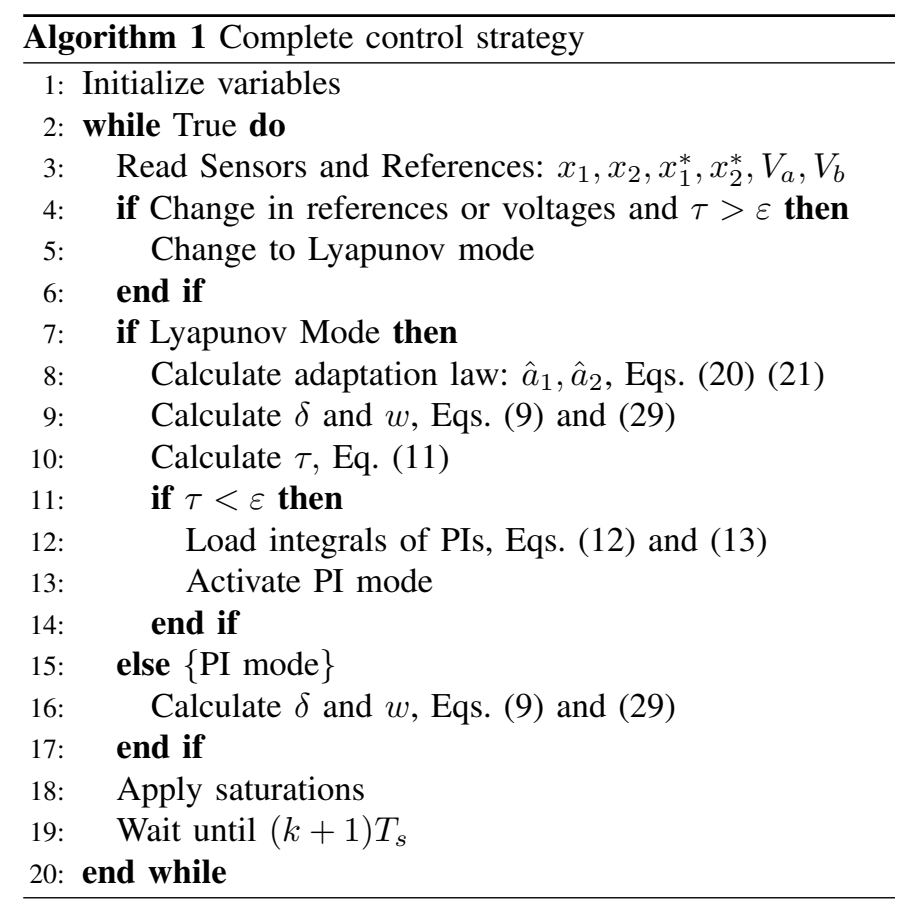

other hand, the design constants allow to regulate the transient behavior although, due to the changing structure of Eqs. (9) and (29), as well as the saturations in $\dot{\delta}$ and $\dot{w}$, the settling time can not be set a priori.

Lastly, it is noteworthy that the expressions of the control proposal are independent on the particular operating point the converter is: it only needs to track the difference between the desired reference and the actual configuration to decide the operating control mode (checking $\tau$ ) and to calculate the inputs $w$ and $\delta$. This is a consequence of Propositions 1 and 2, which prove global asymptotic stability. Therefore, it is not necessary to study whether there are multiple solutions for a given operating point of the converter or it is unique, and no special treatments given the operating point are required.

\section{SiMULATIONS}

This Section describes a series of simulations to evaluate the performance of the control strategy, previous to the implementation in the commercial device. The device has been provided by Epic Power®, so the simulations are adjusted to fit the features of the converter.

A complete model has been created in Simulink®2011b, testing the control strategy directly with the circuit equations (1)-(2). Besides, the control algorithm has been implemented in $C$ language ( $S$-Function Builder [31] [32]), so the code can be directly transferred to a microcontroller or DSP. The model includes the filters to measure $i_{L R}$ and $i_{L I}$, the modulator to generate $u_{1}$ and $u_{2}$ and a worst-case latency of a sampling period $T_{s}$ between the calculation of the action and its application.

To validate the proposal, we compare the results with those obtained by two other control strategies. The first one is based on PIs, which is the existing policy in the device and it is the common alternative in industrial applications [17]. The second one implements a more advanced control, namely, a Sliding 


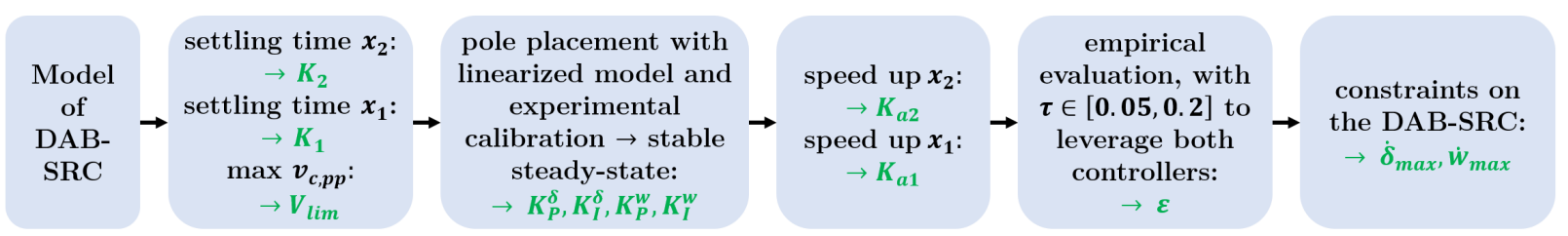

Fig. 4. Flow Chart detailing the design of the control parameters.

Mode Control, taking the state-of-the-art solution proposed in [21]. We have adjusted the values of the control parameters to fit our controller. It is noteworthy that [21] deals with a $\mathrm{DAB}$ converter without resonance, so our problem is harder to solve due to the SRC circuit. Besides, its proposal applies to Single-Input Single-Output systems; therefore, to implement it in our setup we have fixed one of the inputs and used the solution in [21] to control the other one. In particular, we have fixed $\delta=\arctan 2\left(\tilde{x}_{2}, \tilde{x}_{1}\right)$, and apply the Differential Integral Sliding Mode controller from [21] in $w$ to be able to cover the entire dynamic range of the converter. Finally, the Sliding Mode controller includes PIs adjusting the steady-state error since, unlike [21], the sampling frequency is $15-40$ times lower than the switching frequency, making them necessary to avoid oscillations around the desired reference.

It must be underlined that $i_{L I}$ is obtained from the full-wave rectification of $i_{L}$ and its subsequent filtering by an analogue filter. Moreover, to avoid the influence of spurious in $i_{L R}$ and mitigate high frequency noise, a digital filter is included after the measurement to achieve a slight filtering without modifying the hardware. In particular, a first order Butterworth low-pass filter is considered, with a sampling frequency of $T_{s}=2.5 \mathrm{kHz}$ and a normalized cut-off frequency of 0.6

$$
F(z)=\frac{0.5792 z+0.5792}{z+0.1584} \text {. }
$$

This filter is auxiliary and does not belong to the control strategy. The amplitude of the measurement noise has been scaled to fit the one obtained with the real hardware equipment of the experiments on Section VI, which is in the range $[0.25,0.30] \mathrm{V}$ of maximum amplitude. Table I summarizes the main features of the commercial converter.

\begin{tabular}{|c|c|c|}
\hline Parameter & Symbol & Value \\
\hline Sample time & $T_{s}$ & $400 \mu \mathrm{s}$ \\
High bridge voltage & $V_{a}$ & {$[325-400] \mathrm{V}$} \\
Nominal high bridge voltage & $V_{a, n}$ & $375 \mathrm{~V}$ \\
Low bridge voltage & $V_{o}$ & {$[93-138] \mathrm{V}$} \\
Nominal low bridge voltage & $V_{o, n}$ & $116 \mathrm{~V}$ \\
Equivalent coil & $L$ & $110 \mu \mathrm{H}$ \\
Capacitor & $C$ & $254 n \mathrm{~F}$ \\
Equivalent resistance & $R$ & $1 \Omega$ \\
Transformation ratio & $n$ & 2.17 \\
Minimum frequency & $f_{\min }$ & $35 \mathrm{kHz}$ \\
Maximum frequency & $f_{\max }$ & $100 \mathrm{kHz}$ \\
Resonance frequency & $f_{o}$ & $30 \mathrm{kHz}$ \\
Maximum $\delta$ & $\delta_{\max }$ & $\pi / 2 \mathrm{rad}$ \\
Minimum $\delta$ & $\delta_{\min }$ & $-\pi / 2 \mathrm{rad}$ \\
Maximum admissible voltage in $C$ & $V_{\text {lim }}$ & $450 \sqrt{2} \mathrm{~V}$ \\
\hline \multicolumn{2}{|c}{ TABLE I I } \\
MAIN PARAMETERS OF THE CONVERTER.
\end{tabular}

Given the voltage ranges of $V_{a}$ and $V_{o}$ in Table I, 9 combinations are selected to cover all possible scenarios,

\begin{tabular}{|c|c|c|c|c|c|c|c|c|c|}
\hline & $(1)$ & $(2)$ & $(3)$ & $(4)$ & $(5)$ & $(6)$ & $(7)$ & $(8)$ & $(9)$ \\
\hline$V_{a}(\mathrm{~V})$ & 375 & 375 & 375 & 400 & 400 & 400 & 325 & 325 & 325 \\
\hline$V_{o}(\mathrm{~V})$ & 116 & 138 & 93 & 116 & 138 & 93 & 116 & 138 & 93 \\
\hline
\end{tabular}

SimULATED COMBinATIONS OF VOLTAGES $V_{a}$ AND $V_{o}$.

listed in Table II. For each combination, three situations are studied: given a fixed reference value $i_{L R}^{*}=1 \mathrm{~A}$, we apply either positive or negative steps on the reference value $i_{L I}^{*}$ (denoted as $\Delta i_{L I}$ ), always starting from the midpoint of the characterized static range. For each case we record the settling time, checking if $i_{L R}$ goes below $0 \mathrm{~A}$ during the transient for security reasons. These metrics are named $t s_{c p}, t s_{p i}$ and $t s_{s m}$ for our proposal, the PIs and the Sliding Mode respectively.

The control parameters are adjusted to the following: $K_{1}=$ $1, K_{2}=1, K_{P}^{\delta}=0.0002, K_{I}^{\delta}=0.015, K_{P}^{w}=10$, $K_{I}^{w}=5000, K_{a_{1}}=2000, K_{a_{2}}=1000, \dot{\delta}_{\max }=2 \pi / 360 \mathrm{rad}$, $\dot{w}_{\max }=2 \pi 5000 \mathrm{rad} / \mathrm{s}, \varepsilon=0.05$. We compare with two PIs with the following parameters: $K_{P}^{\delta}=0.0001, K_{I}^{\delta}=0.015$, $K_{P}^{w}=1, K_{I}^{w}=1000$. The difference between the PIs of the proposed control strategy and the latest is due to the fact that, in our proposal, the PIs perform in the small signal range, in contrast with the existing control policy which works in the entire dynamic range. Thus, in the existing control policy the PIs must be slow enough to reject the effects of nonlinearities and to evade instability. This is another advantage of the proposal, where the PIs can be tuned to be more demanding since they always work in the small signal range. Besides, the Sliding Mode Controller has been tuned as follows (following the notation from [21]): $K_{1}=50, K_{2}=50, K_{3}=1000$, $\mu=20000, K_{P}^{\delta}=0.0002, K_{I}^{\delta}=0.015, K_{P}^{w}=10$, $K_{I}^{w}=5000$.

The results are shown in Table III. The control strategy achieves a fast dynamic response for all initial and desired configurations, with settling times between $3 \mathrm{~ms}$ and $6 \mathrm{~ms}$. Obtaining such a uniform settling time is an advantage because the convergence time can be known in advance despite the amplitude of the step. Taking into account that $T_{s}=400 \mu \mathrm{s}$, the controller employs $8-15$ samples to drive the converter to the desired configuration, which is the limit usually considered in discrete control theory to capture the relevant information during the transient. In all cases the requirements with regard to $i_{L R}$ are accomplished.

Comparing the static ranges it can be deduced that greater jumps between $V_{a}$ and $V_{o}$ yield to greater static ranges, which is reasonable since there is a greater voltage in the resonant tank. Furthermore, the higher the value of $V_{a}$ and $V_{o}$, the higher the values of $\Delta i_{L I}$. Table III also shows the 


\begin{tabular}{|c|c|c|c|c|c|}
\hline \multirow{2}{*}{ Combination } & \multirow{2}{*}{$i_{L I}(\mathrm{~A})$} & \multirow{2}{*}{$i_{L I}^{*}(\mathrm{~A})$} & \multicolumn{3}{|c|}{ Settling Times (ms) } \\
\cline { 4 - 6 } & & $t s_{c p}$ & $t s_{p i}$ & $t s_{s m}$ \\
\hline$(1)$ & $4.0-8.5$ & 4.75 & 4.0 & 16.0 & 4.0 \\
$(1)$ & $4.0-8.5$ & 7.25 & 3.0 & 10.0 & 3.5 \\
$(1)$ & $4.0-8.5$ & 8.25 & 4.0 & 12.0 & 4.0 \\
\hline$(2)$ & $3.5-6.5$ & 4.0 & 4.0 & 12.0 & 5.0 \\
$(2)$ & $3.5-6.5$ & 5.5 & 3.0 & 10.0 & 3.0 \\
$(2)$ & $3.5-6.5$ & 6.25 & 5.0 & 12.0 & 4.5 \\
\hline$(3)$ & $4.5-9.5$ & 5.5 & 4.0 & 16.0 & 5.0 \\
$(3)$ & $4.5-9.5$ & 8.0 & 4.0 & 10.0 & 4.5 \\
$(3)$ & $4.5-9.5$ & 8.75 & 4.0 & 12.0 & 4.5 \\
\hline$(4)$ & $4.0-10.0$ & 5.0 & 4.0 & 18.0 & 5.0 \\
$(4)$ & $4.0-10.0$ & 7.5 & 3.0 & 8.0 & 3.5 \\
$(4)$ & $4.0-10.0$ & 8.5 & 3.0 & 10.0 & 4.0 \\
\hline$(5)$ & $3.5-7.0$ & 4.0 & 4.0 & 14.0 & 4.5 \\
$(5)$ & $3.5-7.0$ & 5.75 & 3.0 & 10.0 & 3.0 \\
$(5)$ & $3.5-7.0$ & 6.25 & 5.0 & 12.0 & 4.0 \\
\hline$(6)$ & $5.0-11.0$ & 6.0 & 4.0 & 14.0 & 4.0 \\
$(6)$ & $5.0-11.0$ & 9.0 & 4.0 & 6.0 & 4.0 \\
$(6)$ & $5.0-11.0$ & 10.0 & 4.0 & 8.0 & 4.0 \\
\hline$(7)$ & $3.0-6.5$ & 3.5 & 4.0 & 20.0 & 5.0 \\
$(7)$ & $3.0-6.5$ & 5.5 & 5.0 & 10.0 & 5.0 \\
$(7)$ & $3.0-6.5$ & 6.0 & 6.0 & 12.0 & 6.0 \\
\hline$(8)$ & $2.5-5.0$ & 3.0 & 5.0 & 8.0 & 5.0 \\
$(8)$ & $2.5-5.0$ & 4.25 & 4.0 & 8.0 & 4.5 \\
$(8)$ & $2.5-5.0$ & 4.75 & 6.0 & 8.0 & 6.0 \\
\hline$(9)$ & $3.0-8.0$ & 4.0 & 4.0 & 16.0 & 4.5 \\
$(9)$ & $3.0-8.0$ & 6.5 & 4.0 & 10.0 & 4.5 \\
$(9)$ & $3.0-8.0$ & 7.25 & 4.0 & 10.0 & 5.0 \\
\hline & \multicolumn{2}{|c|}{ TABLE III } & & \\
\hline
\end{tabular}

REsults of the Systematic EVALUation of the CONVERTER. RED CELL MEANS A VIOLATION IN THE CONDITION $I_{L R}>0 \mathrm{~A}$

results obtained by the PI control. The settling time is of the order of 2-4 times greater than with our control proposal. In addition, it depends on the amplitude of the step and the specific configuration in which the DAB-SRC is, so it is more complicated to predict. Regarding the Sliding Mode solution, Table III shows that the speed of the transient response is a bit slower than the one achieved by our proposal. The settling time is almost constant as well, but with a very important disadvantage. It can be seen that the red cells marking the cases where the condition $I_{L R}>0 \mathrm{~A}$ is violated appear when we impose a negative step in the $I_{L I}$ reference. This is not allowed in our application. The reason of this is that the Sliding Mode Control requires high control frequencies to be able to correctly approach the sliding surfaces. In our setup, with an appropriate tuning of control parameters, the small control frequency makes the states overpass the sliding frontiers, which in the end make $i_{L R}$ to go beyond $0 \mathrm{~A}$.

To exemplify the behavior of the converter with the control policies, Fig. 5 shows the evolution of the converter against a uniformly random sequence of steps of $i_{L I}$ every $40 \mathrm{~ms}$, with nominal values in $V_{a}$ and $V_{o}$. Thanks to the proposed control strategy, the response is satisfactory covering all the static range of the converter with identical settling times. In the first moments after a reference change, $i_{L R}$ and $i_{L I}$ exhibit some overshoot due to the speed demanded, but always satisfying the operation requirements. The actions are achievable and the adaptation law modifies the values of $\hat{R}$ and $\hat{L}$ (see Fig. 6) to ensure the stability of the system. In this case, the reset of $\hat{R}$ and $\hat{L}$ to the original estimation is not considered but it can be done to guarantee that they prevail near the characterized values. No effect caused by control latency is appreciated.
With the PI policy, the response of the converter is slower than before and is dependent on the amplitude of the step introduced. As $\delta$ hardly changes, $i_{L R}$ does not change either. The current circulating through the transistors is very stable but since the control barely has $\delta$ to regulate $i_{L I}, w$ must take care of regulating the power, with a response in $i_{L I}$ much slower than with the control proposal. This justifies the differences in the response of $i_{L R}$, which influences the transient in $i_{L I}$ and explains the brief overshoot in our control strategy. With the Sliding Mode Control, the transient response is the most spiky in both $i_{L R}$ and $i_{L I}$. In the case of $i_{L R}$ this yields to violations in the condition $i_{L R}>0 \mathrm{~A}$. Besides, a small control frequency with respect to the switching frequency makes the sliding controller to overpass the sliding frontiers between surfaces and, thus, achieve the spiky transient response in $i_{L I}$ as well. As a last remark, the Sliding Mode Control has moderate robustness against uncertainties in the parameters. However, this intrinsic robustness is highly dependent on the particularities of the system and is not quantified, so at some point degradation or uncertainty may destabilize the converter. On the other side, our proposal has theoretical guarantees of robustness due to the adaptive law and global asymptotic stability is irrespective of uncertainty.

The advantages and disadvantages of our proposal, compared to the other techniques, are summarized in Table IV. Green color is advantage, red color is disadvantage, and yellow is neutral.

\section{EXPERIMENTS}

The setup of the experiments is shown in Fig. 7. The converter is disassembled, with access to the motherboard, the microcontroller and the auxiliary measuring devices. The oscilloscope is a model MSO7104B of InfiniiVision ${ }^{\circledR}[33]$ and has four channels and a bandwidth of $1 \mathrm{GHz}$. The oscilloscope is over a bank of power supplies, which provide the energy to the converter. Specifically, these power supplies can set the voltage for bi-directional current demands, so they can supply energy (load) or recover it (discharge). The 32-bit microcontroller that implements the control strategy comes with floating point unit (FPU) and can perform the arctan 2 operation [34]. It also has enough configurable output pins to monitor, either through serial communication or direct measurement with an oscilloscope, internal variables for debugging.

The results of the experiments are shown in Figs. 8-10. The first series of experiments, in Fig. 8, consists in applying reference steps of different amplitude (positive and negative) with different voltages and starting up conditions. Fig. 8 shows that the control strategy achieves a fast and stable response even when the converter is turned on. In relation with $i_{L R}$, the microcontroller receives a data each $10 \mathrm{~ms}$ so it is not possible to follow its transient response. However, we have verified that after $10 \mathrm{~ms}$ from the change in the reference, $i_{L R}$ converges to $i_{L R}^{*}$, a hundred times faster with our control solution than with the previous policy. The experiments validate that the control strategy globally achieves a fast and stable response, converging to the desired configuration in not more than $6 \mathrm{~ms}$. It is noteworthy that in Fig. 8c and Fig. 8e a spike appears in 
Simulation results

Evolution of $i_{L I}$ over time

Our proposal
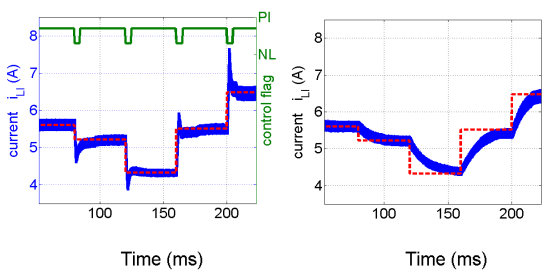

Evolution of $\delta$ over time

Our proposal

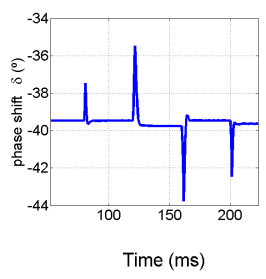

PIs

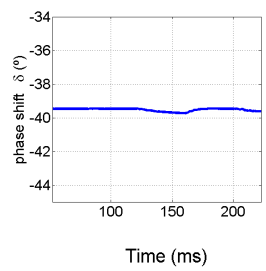

Sliding Mode Control

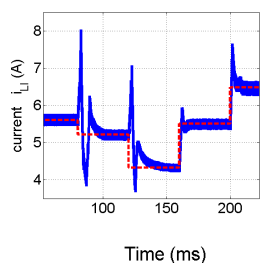

Sliding Mode Control

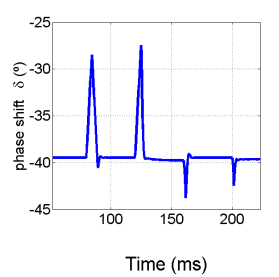

Evolution of $i_{L R}$ over time

PIs

Sliding Mode Control
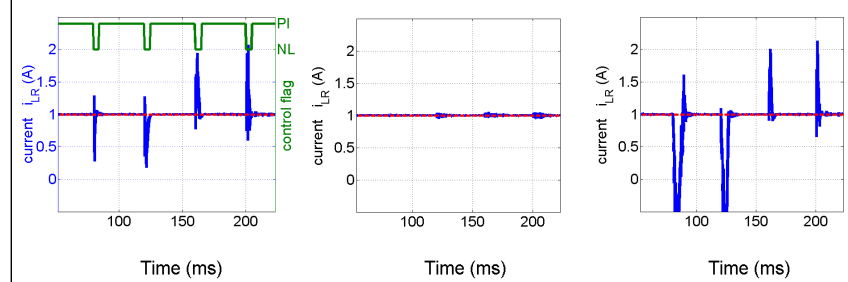

Evolution of $f=w / 2 \pi$ over time

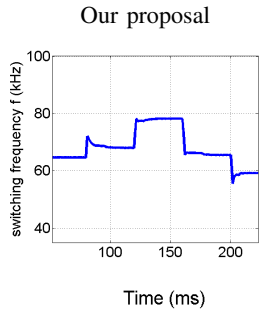

PIs

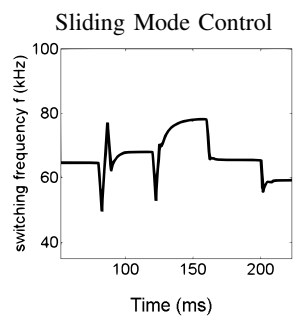

Fig. 5. Behavior of the converter against a uniformly random sequence of steps in $i_{L I}^{*}$, where $V_{a}=V_{a, n}$ and $V_{o}=V_{o, n}$. Green flag indicates the operation mode of our control strategy. PI label means PI mode and NL label means Nonlinear mode.

\begin{tabular}{|c|c|c|}
\hline Our proposal & PI Control & Sliding Mode Control \\
\hline Global Asymptotic Stability & Small Gain Stability & Global Asymptotic Stability \\
\hline Constant Fast Settling Time & Step-dependent Slow Settling Time & $\begin{array}{c}\text { Constant Fast (less than our proposal) Settling } \\
\text { Time }\end{array}$ \\
\hline \multirow{3}{*}{$\begin{array}{c}\text { Overshoot may appear } \\
\text { Preserves } i_{L R}>0 \mathrm{~A} \\
\text { Adaptation adds robustness against uncertainty } \\
\text { and keeps fast transient }\end{array}$} & No overshoot & Overshoot may appear \\
\hline & Preserves $i_{L R}>0 \mathrm{~A}$ & Does not preserve $i_{L R}>0 \mathrm{~A}$ \\
\hline & Dynamics must be slowed down for robustness & $\begin{array}{c}\text { Sliding is robust against moderate levels of } \\
\text { uncertainty }\end{array}$ \\
\hline
\end{tabular}

TABLE IV

COMPARISON AMONG CONTROLLERS. GREEN COLOR IS ADVANTAGE, RED COLOR IS DISADVANTAGE, AND YELLOW IS NEUTRAL.

Evolution of estimates during simulation
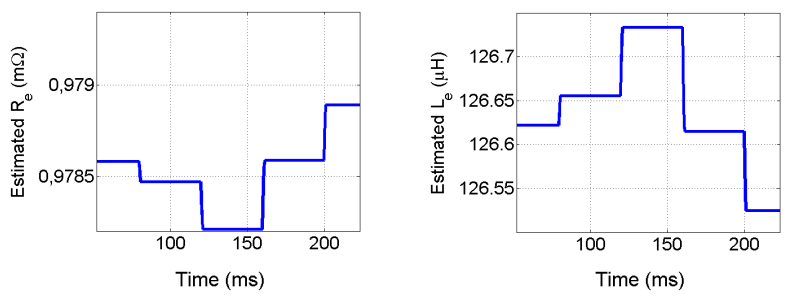

Fig. 6. Evolution of the estimation of $R$ and $L$.

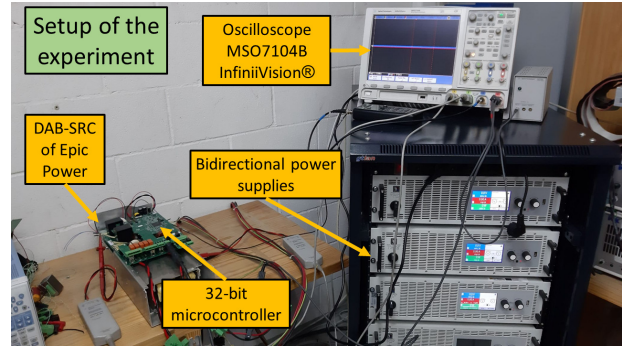

Fig. 7. Setup of the experiment.

the reference change. This is due to the influence of the output filter of the converter when it is started up. By construction of the commercial converter and for security reasons, the PWM lasts one sample time to apply the control inputs, so the first $400 \mu$ s the converter is alone against the predefined values of $w$ and $\delta$, resonating with the $4.5 \mathrm{kHz}$ frequency of the output filter. After that, the control is applied and the spike disappears.

To further investigate our proposal, the next experiment evaluates the response of the converter against uncertainties in $R$ and $L$, recalling that our proposal is immune to the value of $C$. To do so, we have mapped the uncertainty by initializing the control with values of $R$ and $L$ different from those characterized. In particular, we have assumed differences of the $50 \%$, which is much higher than the typical deviation of these parameters. The experiments have been conducted with the converter operating at $V_{a}=375 \mathrm{~V}, V_{o}=116 \mathrm{~V}, i_{L I}^{*}$ from $7 \mathrm{~A}$ to $11 \mathrm{~A}$. As we can see in Fig. 9, the transient and steadystate response is almost mirrored irrespective of the uncertainty in $R$ and $L$. This is an evidence of the robustness achieved by the adaptation law of our proposal. In all the cases the control obtains zero steady-state error.

The final experiment assesses the robustness of the control strategy against sudden changes in $V_{a}$ and $V_{o}$. We have programmed a sequence of random uniformly random steps in $V_{a}$ and $V_{o}$, positive or negative, each $40 \mathrm{~ms}$. The delay between steps in $V_{a}$ and $V_{o}$ is also random. The converter has been driven to steady-state at $V_{a}=375 \mathrm{~V}, V_{o}=116 \mathrm{~V}$, and $I_{L I}=3.1 \mathrm{~A}$. Fig. 10 shows that the converter is completely 


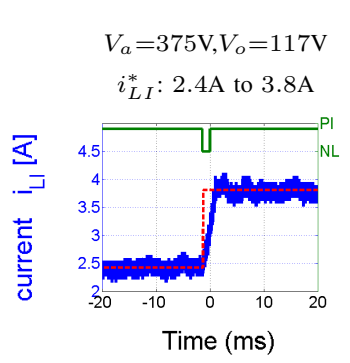

Experimental results: converter against different values of voltage and current.
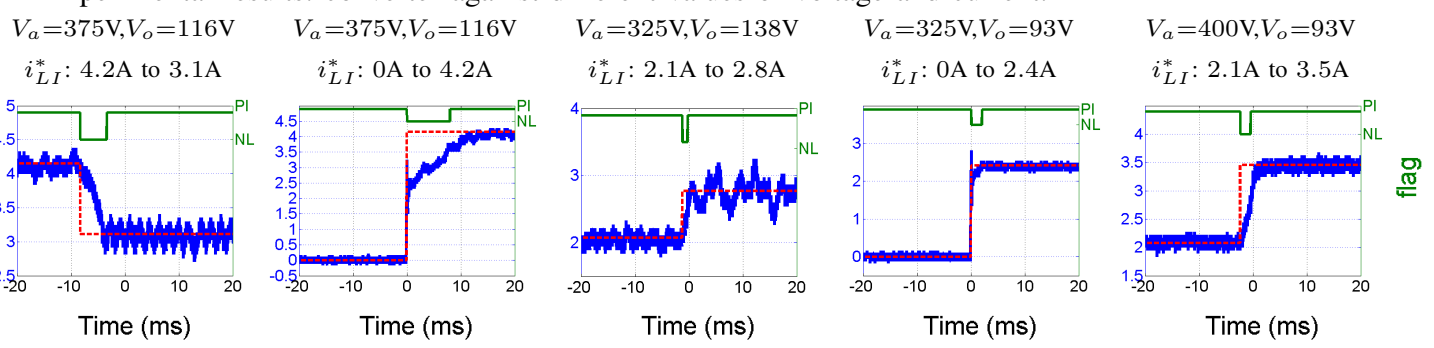

Fig. 8. Results of the experiment with the commercial converter against different values of voltage and current. All of them have been conducted using the nominal parameters of the converter. Green flag indicates the operation mode. PI label means PI mode and NL label means Nonlinear mode.

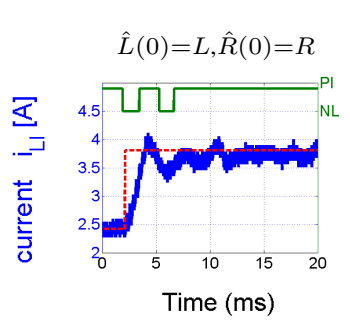

Experimental results: response of the converter against uncertainty in the parameters.
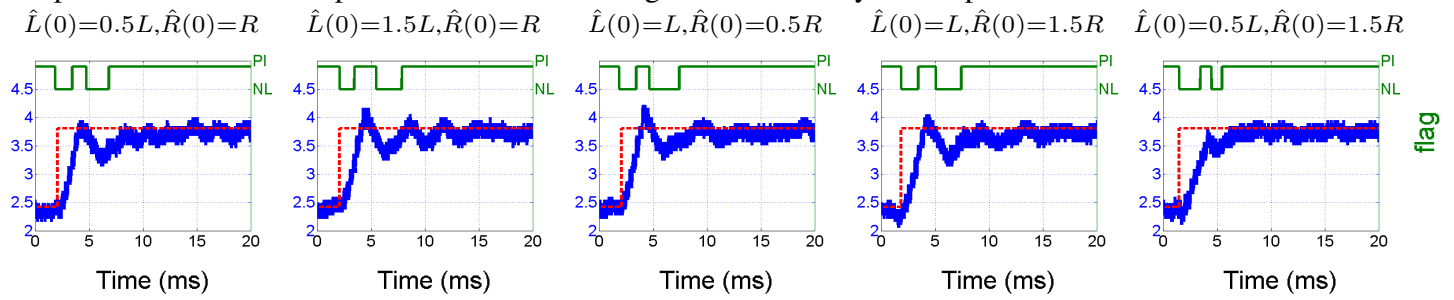

Fig. 9. Results of the experiment with the commercial converter against uncertainty in the parameters. All of them have been conducted with the converter operating at $V_{a}=375 \mathrm{~V}, V_{o}=116 \mathrm{~V}, i_{L I}^{*}$ from $2.4 \mathrm{~A}$ to $3.8 \mathrm{~A}$. Green flag indicates the operation mode. PI label means PI mode and NL label means Nonlinear mode.

Experimental results: robustness of the converter against disturbances on $V_{a}$ and $V_{o}$.
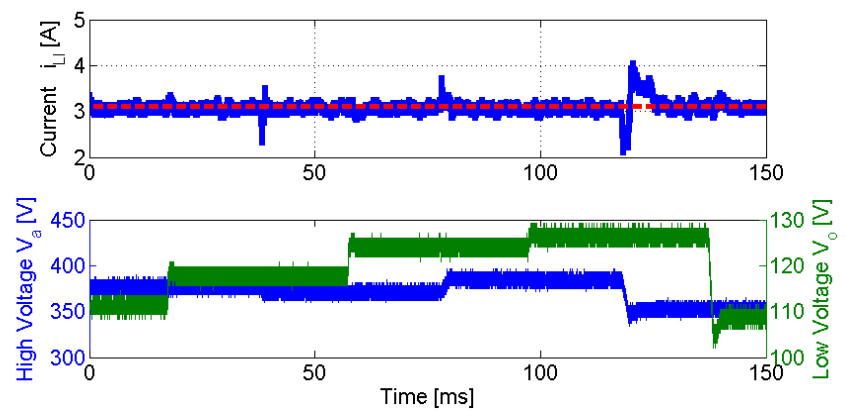

Fig. 10. Results of the experiment with the commercial converter against disturbances on $V_{a}$ and $V_{o}$ : (top) evolution of $i_{L I}$; (bottom) disturbances in $V_{a}$ (blue) and $V_{o}$ (green). The converter has been firstly driven to $V_{a}=375 \mathrm{~V}, V_{o}=116 \mathrm{~V}$, and $I_{L I}=3.1 \mathrm{~A}$

robust against sudden changes in the voltages at both sides. The worst case, around $t=120 \mathrm{~ms}$, corrects $I_{L I}$ in less than $5 \mathrm{~ms}$ and corresponds to a high-amplitude step in $V_{a}$. The rest of the time $I_{L I}$ follows the reference irrespective of the disturbances.

\section{CONCLUSIONS}

This paper has presented a novel control strategy for a SRC in a DAB topology based on nonlinear control techniques. It has been shown how this design achieves desirable properties from the application point of view. The control law ensures the global asymptotic stability of the converter which yields to a substantial improvement in the speed of the response, as there is no risk of instability. Besides, modifications have been made to the initial control solution to ease the integration into a real prototype without affecting its stability properties. The complete strategy maintains the benefits of the nonlinear control law and improves the performance with respect to existing control loops. Moreover, its benefits have been validated by several simulations and experiments with a commercial converter.

\section{REFERENCES}

[1] Z. Pavlović, J. A. Oliver, P. Alou, O. García, and J. A. Cobos, "Bidirectional dual active bridge series resonant converter with pulse modulation," in IEEE Applied Power Electronics Conference and Exposition, pp. 503-508, Feb. 2012.

[2] B. Zhao, G. Wang, and W. G. Hurley, "Analysis and performance of LCLC resonant converters for high-voltage high-frequency applications," IEEE Journal of Emerging and Selected Topics in Power Electronics, vol. 5, no. 3, pp. 1272-1286, Sep. 2017.

[3] E. Oyarbide, I. Elizondo, A. Martínez-Iturbe, C. Bernal, and J. Irisarri, "Ultracapacitor-based plug play energy-recovery system for elevator retrofit," in IEEE International Symposium on Industrial Electronics, pp. 462-467, Jun. 2011.

[4] C. Buccella, C. Cecati, and H. Latafat, "Digital control of power converters-a survey," IEEE Transactions on Industrial Informatics, vol. 8, no. 3, pp. 437-447, Aug. 2012.

[5] J. A. Baroudi, V. Dinavahi, and A. M. Knight, "A review of power converter topologies for wind generators," Renewable Energy, vol. 32, no. 14, pp. 2369-2385, 2007.

[6] R. Tavakoli and Z. Pantic, "Analysis, design, and demonstration of a 25$\mathrm{kW}$ dynamic wireless charging system for roadway electric vehicles," IEEE Journal of Emerging and Selected Topics in Power Electronics, vol. 6, no. 3, pp. 1378-1393, Sep. 2018.

[7] R. W. De Doncker, D. M. Divan, and M. H. Kheraluwala, "A three-phase soft-switched high-power-density DC/DC converter for high-power applications," IEEE Transactions on Industry Applications, vol. 27, no. 1, pp. 63-73, 1991. 
[8] M. Kheraluwala, R. W. Gascoigne, D. M. Divan, and E. D. Baumann, "Performance characterization of a high-power dual active bridge DCto-DC converter," IEEE Transactions on Industry Applications, vol. 28, no. 6, pp. 1294-1301, 1992.

[9] V. Vorperian and S. Cuk, "A complete dc analysis of the series resonant converter," in 1982 IEEE Power Electronics Specialists conference, pp. 85-100. IEEE, 1982.

[10] R. Oruganti and F. C. Lee, "Resonant power processors, part i-state plane analysis," IEEE Transactions on industry applications, no. 6, pp. $1453-1460,1985$

[11] R. W. Erickson and D. Maksimovic, Fundamentals of power electronics. Springer Science \& Business Media, 2007.

[12] F. Krismer and J. W. Kolar, "Accurate power loss model derivation of a high-current dual active bridge converter for an automotive application," IEEE Transactions on Industrial Electronics, vol. 57, no. 3, pp. 881-891, 2009.

[13] X. Li and A. K. Bhat, "Analysis and design of high-frequency isolated dual-bridge series resonant DC/DC converter," IEEE Transactions on Power Electronics, vol. 25, no. 4, pp. 850-862, 2009.

[14] F. Vasca and L. Iannelli, Dynamics and Control of Switched Electronic Systems Advanced Perspectives for Modeling, Simulation and Control of Power Converters., 1st ed. Springer, London, 2012.

[15] D. Seltzer, L. Corradini, D. Bloomquist, R. Zane, and D. Maksimović, "Small signal phasor modeling of dual active bridge series resonant DC/DC converters with multi-angle phase shift modulation," in IEEE Energy Conversion Congress and Exposition, Sep. 2011.

[16] D. Seltzer, D. Bloomquist, R. Zane, and D. Maksimovic, "Gainscheduled control of multi angle phase shift modulated dual active bridge series resonant DC/DC converters," in IEEE Workshop on Control and Modeling for Power Electronics, pp. 1-7, 2012.

[17] S. P. Engel, N. Soltau, H. Stagge, and R. W. De Doncker, "Dynamic and balanced control of three-phase high-power dual-active bridge DCDC converters in DC-grid applications," IEEE Transactions on Power Electronics, vol. 28, no. 4, pp. 1880-1889, 2012.

[18] C. Zhang, J. Wang, S. Li, B. Wu, and C. Qian, "Robust control for PWMbased DC-DC buck power converters with uncertainty via sampled-data output feedback," IEEE Transactions on Power Electronics, vol. 30, no. 1, pp. 504-515, Jan. 2015.

[19] C. Meza, D. Biel, D. Jeltsema, and J. M. A. Scherpen, "Lyapunov-based control scheme for single-phase grid-connected PV central inverters," IEEE Transactions on Control Systems Technology, vol. 20, no. 2, pp. 520-529, Mar. 2012.

[20] J.-J. E. Slotine, W. Li et al., Applied nonlinear control, vol. 199, no. 1. Prentice Hall Englewood Cliffs, NJ, 1991.

[21] Y.-C. Jeung and D.-C. Lee, "Voltage and current regulations of bidirectional isolated dual-active-bridge DC-DC converters based on a doubleintegral sliding mode control," IEEE Transactions on Power Electronics, vol. 34, no. 7, pp. 6937-6946, 2018.

[22] S. Saggini, W. Stefanutti, E. Tedeschi, and P. Mattavelli, "Digital deadbeat control tuning for DC-DC converters using error correlation," IEEE Transactions on Power Electronics, vol. 22, no. 4, pp. 1566-1570, 2007.

[23] P. Cortés, M. P. Kazmierkowski, R. M. Kennel, D. E. Quevedo, and J. Rodríguez, "Predictive control in power electronics and drives," IEEE Transactions on industrial electronics, vol. 55, no. 12, pp. 4312-4324, 2008.

[24] J. Rodríguez and P. Cortés, Predictive control of power converters and electrical drives, vol. 40. John Wiley \& Sons, 2012.

[25] R. Oruganti and F. C. Lee, "Resonant power processors, part ii-methods of control," IEEE Transactions on Industry Applications, no. 6, pp. $1461-1471,1985$.

[26] R. Oruganti, J. J. Yang, and F. C. Lee, "Implementation of optimal trajectory control of series resonant converter," IEEE Transactions on Power Electronics, vol. 3, no. 3, pp. 318-327, 1988.

[27] W. Feng, F. C. Lee, and P. Mattavelli, "Simplified optimal trajectory control (SOTC) for LLC resonant converters," IEEE Transactions on Power Electronics, vol. 28, no. 5, pp. 2415-2426, 2012.

[28] S. R. Sanders, J. M. Noworolski, X. Z. Liu, and G. C. Verghese, "Generalized averaging method for power conversion circuits," IEEE Transactions on Power Electronics, vol. 6, no. 2, pp. 251-259, 1991.

[29] J. Mahdavi, A. Emaadi, M. D. Bellar, and M. Ehsani, "Analysis of power electronic converters using the generalized state-space averaging approach," IEEE Transactions on Circuits and Systems I: Fundamental Theory and Applications, vol. 44, no. 8, pp. 767-770, 1997.

[32] MathWorks $®$, Simulink®User's Guide. MathWorks $® 2020$.
[30] H. K. Khalil, Nonlinear Control. Pearson Education, 2014.

[31] MathWorks®, Simulink®Developing S-Functions. MathWorks®, 2020.

[33] A. Technologies, "Agilent InfiniiVision 7000B Series Oscilloscopes user's guide," 2010.

[34] T. Instruments, "CLA math library user guide," 2018.

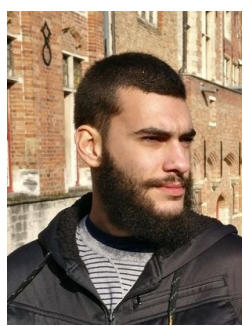

Eduardo Sebastián (S'21) received the B.Eng. in Electronic and Automatic Engineering (Hons) and the M.Eng. in Electronics (Hons) from the University of Zaragoza in 2019 and 2020 respectively. He is currently a Ph.D. Candidate in the Department of Computer Science and Systems Engineering at the University of Zaragoza, funded by a FPU national grant (1st rank). His current research interests include nonlinear control, distributed systems, power electronic converters and multi-robot systems.

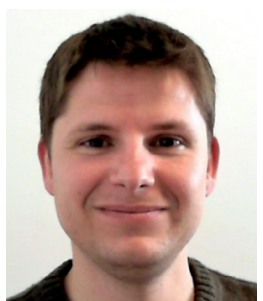

Eduardo Montijano (M'12) is an Associate Professor in the Departamento de Informática e Ingeniería de Sistemas at Universidad de Zaragoza in Spain. He received the M.Sc. and Ph.D. degrees from the Universidad de Zaragoza, Spain, in 2008 and 2012 respectively. $\mathrm{He}$ was a faculty member at Centro Universitario de la Defensa, Zaragoza, between 2012 and 2016. His main research interests include distributed algorithms and automatic control in perception problems. His Ph.D. obtained the extraordinary award of the Universidad de Zaragoza in the 2012-2013 academic year.

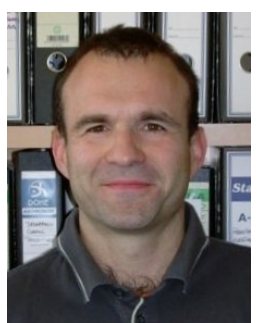

Estanis Oyarbide was born in Beasain, Spain, on June 30, 1969. He received the B.Eng. degree from the University of Mondragon, Mondragon, Spain, in 1992, and the M.Eng. and Ph.D. degrees from the National Polytechnic Institute of Grenoble, Grenoble, France, in 1994 and 1998, respectively. From 1998 to 2002 he was a Lecturer in the Department of Electronics, University of Mondragon, specializing in power converters and adjustable speed drives. $\mathrm{He}$ is currently a Lecturer in the Department of Electronic Engineering and Communications and a Researcher at the Aragon Institute for Engineering Research, both at the University of Zaragoza, Zaragoza, Spain, which he joined in 2002. His current research interests include high efficiency power conversion.

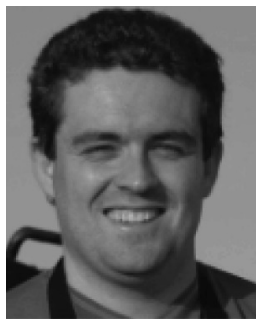

Carlos Bernal (Member, IEEE) received the M.Sc. and Ph.D. degrees in electrical engineering from the University of Zaragoza, Spain, in 2000 and 2012, respectively. Since 2001, he has been an Associate Professor, with special interest in power and mixed-signal electronics, with the University of Zaragoza. His research interest is focused on high-efficiency resonant switch-mode converters and their control.

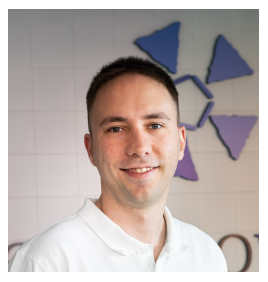

Rubén Gálvez was born in Zaragoza, Spain, on February 18,1988 . He received the B.Eng. and M.Eng degree from the University of Zaragoza, Zaragoza, Spain in 2010 and 2011, respectively. From 2011 to 2013, he was a researcher in the Group of Power Electronics and Microelectronics of the University of Zaragoza, specializing in power converters and adjustable speed drives. He is currently the Technical Manager of Epic Power Converters and Ph.D student in the Aragon Institute for Engineering Research,

at the University of Zaragoza, Zaragoza, Spain. The Ph.D research is related to high efficiency DC power conversion. 